\title{
Solving of the Transcendental Equations in Layered Media
}

\author{
Neng-zhong Lei, ${ }^{1, a} \&$ le-Lung Chung ${ }^{1, b^{*}}$ \\ ${ }^{1}$ Department of Civil Engineering and Architecture, Wuyi University, Fujian 354300, China \\ ${ }^{2}$ Department of Civil Engineering and Architecture, Wuyi University, Fujian 354300, China \\ a1961808133@qq.com, ${ }^{\mathrm{b}} 1091358613 @ q q . c o m$
}

Keywords: Wave propagation, Impedance matrix, Dynamic stiffness and Soil-structure Interaction. Abstract. In this paper, we use an efficient method to solve the wave numbers of transient wave propagation in layered media. To solve those problems, the analytic solution for the interior domain is combined both a homogeneous solution and a particular solution, the exterior domain is described by a homogeneous solution only. To obtain the homogeneous solution, one has challenge to solve the complex root of the transcendental equations will be discussed. For the soil-structure interaction problem, the wave number of transient wave propagation in layered media will be used for the impedance matrix of embedded axial symmetric foundation.

\section{Introduction}

From Soil-structure interaction problems, many approaches may be considered to deal with the soil-structure interaction analysis problems. In the early time, the finite element method had been popular to determine the impedance functions for foundation embedded in an elastic stratum. In this model, the soil medium could be divided into near-field and far-field. For the near-field, regular meshing of finite element method was employed. Finite element method was used for the near-field [1], there were many approach methods, such as viscous boundary [2,3], transmitting boundary [4], boundary element method [5], and infinite element methods [6]. Among the above mentioned modelling, boundary element method requires boundary discretization which could be reduced some computational cost while compared to that of finite element method. In boundary element method, Green function was used as a fundamental solution to generate the impedance functions at the assumed boundary of structure [7].

Sezawa [8] has developed a procedure to separate the dilatational and the rotational waves to solve the wave equation in cylindrical coordinates for the half-space medium. Tzong and Penzien [1] extended this solution to find impedance matrix of a single-layer half-space system. Regards of analytical solution technique, Liou [9] successfully finds the shapes function of the stress and displacement fields which are prescribed harmonic displacement time history in interior domain.

Liou has developed a technique to decompose the boundary conditions to fit the general solutions of wave equations in cylindrical coordinates for the cases of layered media. The technique has been successfully applied to find the impedance functions for foundations on layered half-space medium [10] and axial symmetric foundation embedded in layered medium [11]. Liou and Chung [12] extended the methods which are prescribed harmonic tractions time history to found the shapes function of the stress and displacement fields in interior domain. Recently, Ding et al [13] use iteration process to find the roots of the transcendental equation in layered soil by using Laplace transformation method. Popov and Vaysfel'd [14] calculate the real roots of the transcendental equation by using residue theorem in the conical layered elastic cone. Slavica et al [15] find an exact analytical closed-form solution of some families of transcendental equations by using the Special Trans Functions Theory (STFT).

In this paper, we will employ the analytic solution for soil-structure interaction in layered media to developing a general-purpose program of the transcendental equations. In developing the numerical problem, the Love modes of the transient wave propagation problems in layered media will be analytical predicted of the wave numbers by using numerical methods. Consequently, the wave numbers of transcendental equations for soil-structure interaction in layered media will be derived analytically and numerically. 


\section{Analytical derivations for three-dimensional wave propagation problems}

The total system is shown in Figure 1. The whole soil domain is divided into interior and exterior domain. The general equation of wave propagation for the homogeneous solution was derived by Liou and Chung [12], all the results of the homogeneous solutions are shown in Table 1.

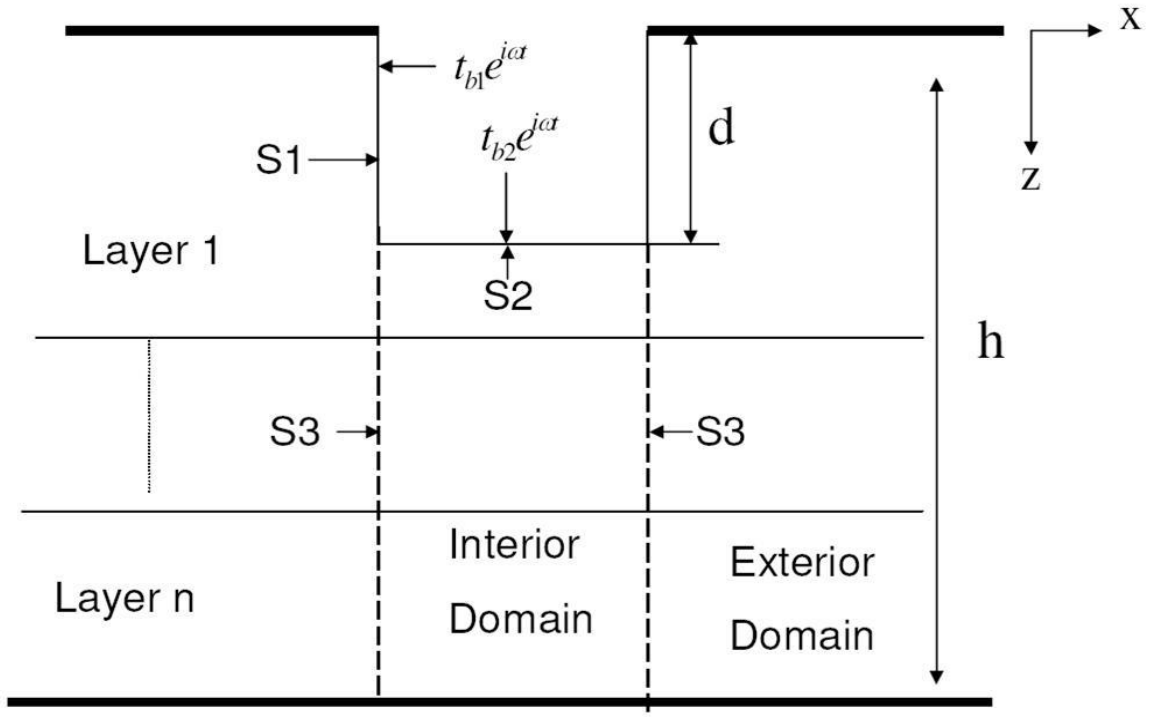

Rigid bedrock

Fig. 1. Total Soil System With Prescribed Tractions.

Table 1 The homogeneous solutions of the complex roots equations.

\begin{tabular}{|c|c|c|c|}
\hline \multicolumn{4}{|c|}{ Complex wave number } \\
\hline \multirow{4}{*}{$\begin{array}{l}\text { Basic } \\
\text { equations }\end{array}$} & \multirow{2}{*}{$\begin{array}{l}\text { For } \\
\text { Love } \\
\text { mode }\end{array}$} & $\begin{array}{l}\text { Exterior } \\
\text { domain }\end{array}$ & $t_{55}=0$ \\
\hline & & $\begin{array}{l}\text { Interior } \\
\text { domain }\end{array}$ & $t_{56}=0$ \\
\hline & \multirow{2}{*}{$\begin{array}{c}\text { For } \\
\text { Rayleigh } \\
\text { mode }\end{array}$} & $\begin{array}{l}\text { Exterior } \\
\text { domain }\end{array}$ & $t_{11} \times t_{22}-t_{12} \times t_{21}=0$ \\
\hline & & $\begin{array}{l}\text { Interior } \\
\text { domain }\end{array}$ & $t_{13} \times t_{24}-t_{14} \times t_{23}=0$ \\
\hline
\end{tabular}

Form the Table 1, it is easily to analyze the wave number of Love mode. The wave number k's are responding to the exterior domains of the complex roots equations. For the case of the wave number $\mathrm{k}$ is representing Love modes, we obtain

$$
t_{55}=\operatorname{Cosh}\left[v^{\prime} h\right]=0
$$


where $k$ is the wave number of the mode, $v=\sqrt{k^{2}\left(\frac{\omega^{2}}{C_{P}^{2}}\right)}, v^{\prime}=\sqrt{k^{2}\left(\frac{\omega^{2}}{C_{S}^{2}}\right)}, C_{S}$ is the shear wave velocity and $C_{P}$ is the compressional wave velocity, $\omega$ is natural frequency. Methods to solving the wave problem from the Eq.(1) can be expressed as

$$
\operatorname{Cosh}\left[v^{\prime} h\right]=\operatorname{Cos}\left[i v^{\prime} h\right]=0
$$

The wave number $k$ can be determined as

$$
k_{N}= \pm \sqrt{\frac{\omega^{2}}{C_{S}^{2}} \frac{\pi^{2}(N+0.5)^{2}}{h^{2}}}, N=0,1,2,3 \cdots
$$

Where $C_{S}=\sqrt{\frac{G}{\rho}}$ and $G=1+2 \xi i$. From the Eq.(3), we can find the wave numbers of love modes have a pair of conjugate the roots.

For the case of the wave number $k$ is representing Rayleigh modes, we obtain

$$
t_{11} t_{22}-t_{12} t_{21}=0
$$

From the Eq.(4), they are multiplied by a lot of transcendental functions. Thus, we will use a numerical method to solve the wave numbers of transient wave propagation in layered media. Due to Eqs. (1) and (4) have an infinite number of roots, all the roots must satisfying the radiation condition in exterior domain. As $r \rightarrow \infty, H_{n}^{(2)}(k r)$ is the Hankel function of the second kind of order $n$. It can be expressed as asymptotic form:

$$
H_{n}^{(2)}(k r) \approx\left(\frac{2}{\pi k r}\right) \exp \left(i k r+\frac{n \pi}{2} \quad \frac{\pi}{4}\right)\left[1+\frac{4 n^{2} 1}{1 !(8 i k r)}+\cdots\right]
$$

If $k=A+i B$ is an eigenvalue with eigenvector, then $\bar{k}$ is another pair of conjugate the roots $r$. In order to satisfying the radiation condition in Eq.(5), we should choose the wave number $k$ which is either $A \geq 0$ or $B<0$.

\section{Root searching scheme for transcendental equations}

Eqs. (1) and (4) have an infinite number of roots on the complex plane. In order to solve the wave numbers of transient wave propagation in layered media, the transcendental function can be expressed as

$$
f(z)=\Phi(x, y)+i \Psi(x, y)
$$

Where $z=x+i y$ is a complex variable, $\Phi(x, y)$ and $\Psi(x, y)$ are the real and the imaginary parts of $f(z)$. The scheme is to find the approximate $z$ such that

$$
f(z)=\Phi(x, y)+i \Psi(x, y) \approx 0+i 0
$$

To find all the approximate root's, one needs to divide the region into the mesh shown in Figure 2. 


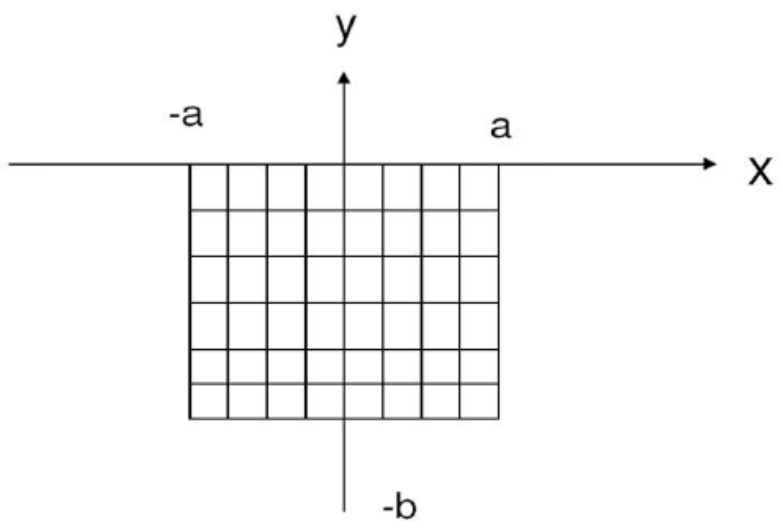

Fig. 2. Mesh of the region $-\mathrm{a} \leq \mathrm{x} \leq \mathrm{a}$ and $-\mathrm{b} \leq \mathrm{y} \leq \mathrm{b}$ on complex plane.

Meanwhile, Figure 3 show the complex root in the typical grid pattern of both $\Phi(x, y)=0$ and $\Psi(x, y)=0$, where the $z 1$ to $z 4$ are four points of the typical grid pattern.

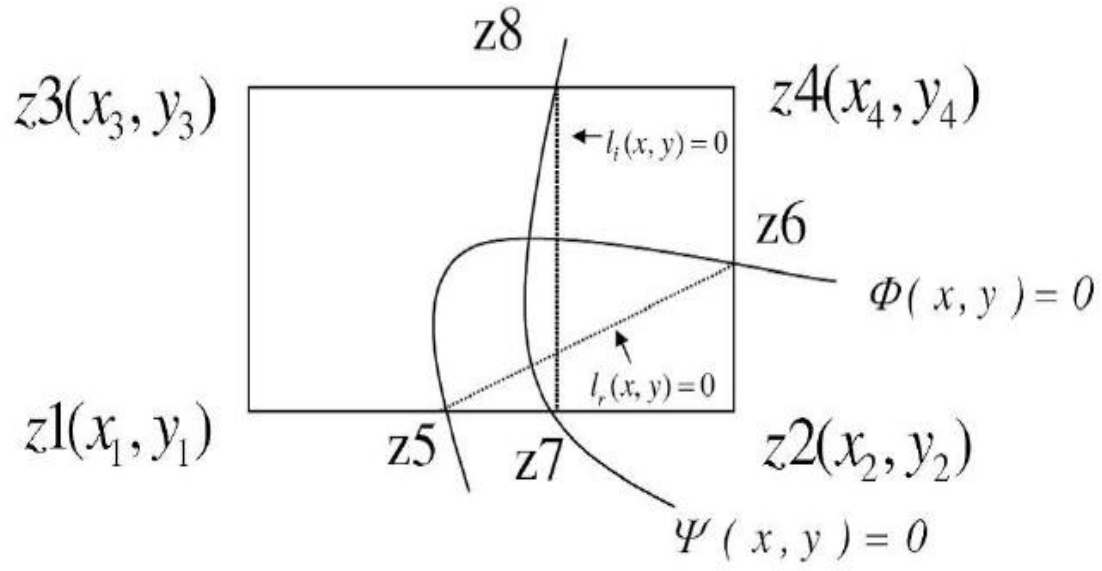

Fig. 3. Typical grid patterns.

If the line of $\Phi(x, y)=0$ goes through a small grid pattern, we have the result of $\Phi(z 1) \Phi(z 2) \leq 0$. Otherwise, the line of $\Phi(x, y)=0$ is not contain. By the similar way, we have the line $\Psi(x, y)=0$ which is $\Psi(z 1) \Psi(z 2) \leq 0$. Thus, we have found a small grid pattern in both $\Phi(x, y)=0$ and $\Psi(x, y)=0$. If any two function values at the four corners of the grid have different signs. To determine the root above two functions, we have two linear equations:

$$
\begin{aligned}
& l_{r}(x, y)=a_{1} x+b_{1} y+c_{1}=0 \\
& l_{i}(x, y)=a_{2} x+b_{2} y+c_{2}=0
\end{aligned}
$$

Where $a_{1}=y_{5}-y_{6}, b_{1}=x_{6}-x_{5}, c_{1}=x_{5} y_{6}-x_{6} y_{5}, a_{2}=y_{7}-y_{8}, b_{2}=x_{8}-x_{7}$ and $c_{2}=x_{7} y_{8}-x_{8} y_{7}$, to determine the root in the grid, the approximate root is

$$
z_{n}=x_{n}+i y_{n}
$$


Where $y_{n}=\frac{c_{1} a_{2}-c_{2} a_{1}}{b_{2} a_{1}-b_{1} a_{2}}, \quad x_{n}=\frac{\left(b_{1} y_{n}+c_{1}\right)}{a_{1}}$, if $a_{1} \geq a_{2}, x_{n}=-\frac{\left(b_{2} y_{n}+c_{2}\right)}{a_{2}}$, if $a_{2} \geq a_{1}$.

\section{Numerical investigations}

In this numerical study, a rigid massless circular plate resting on a single layer stratum is shown in Figure 4.

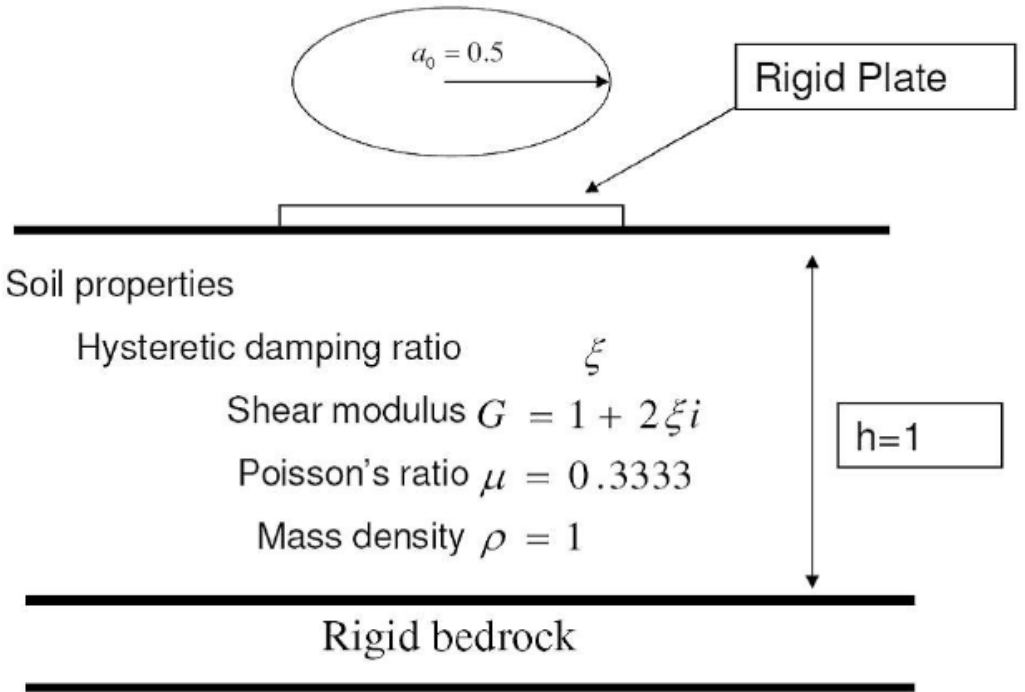

Fig. 4. Soil profile of example.

Form Love mode of Eq.(1), we can find out the analytical solution of the wave number $k$ can be determined from the Eq.(3), the Figs. $5 \sim 7$ are show $\omega=0.4, \omega=2$ and $\omega=6$ of an analytical wave numbers of undamping system of real part $\mathrm{k}$ versus imaginary part $\overline{\mathrm{k}}$ in the complex plane. Form the Figure 5 , when $N=0,1,2 \cdots$ are show the position of analytical complex wave numbers, we can find that the analytical complex wave numbers of $k_{N}$ only exist in imaginary axial and $\bar{k}_{N}$ is another pair of the roots.

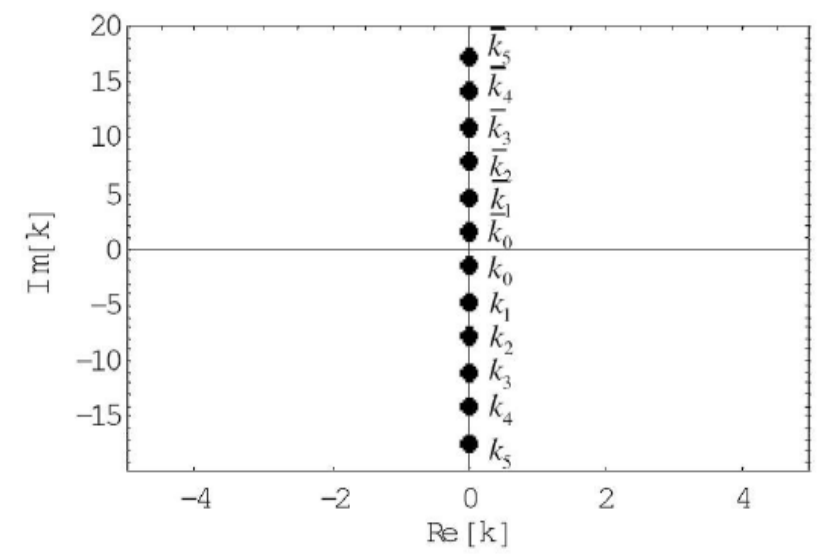

Fig.5 Analytical wave number values of undamping system at $\omega=0.4$.

In the Figures 6 and 7 are show when the natural frequency is increasing, the analytical complex wave numbers from imaginary axial close to real axial. In the high frequency, it is obviously that those complex wave numbers have real part at the low order of $N$, the contribution of the low order wave numbers form the Love modes is most important about finding the impedance matrices in layered medium. 

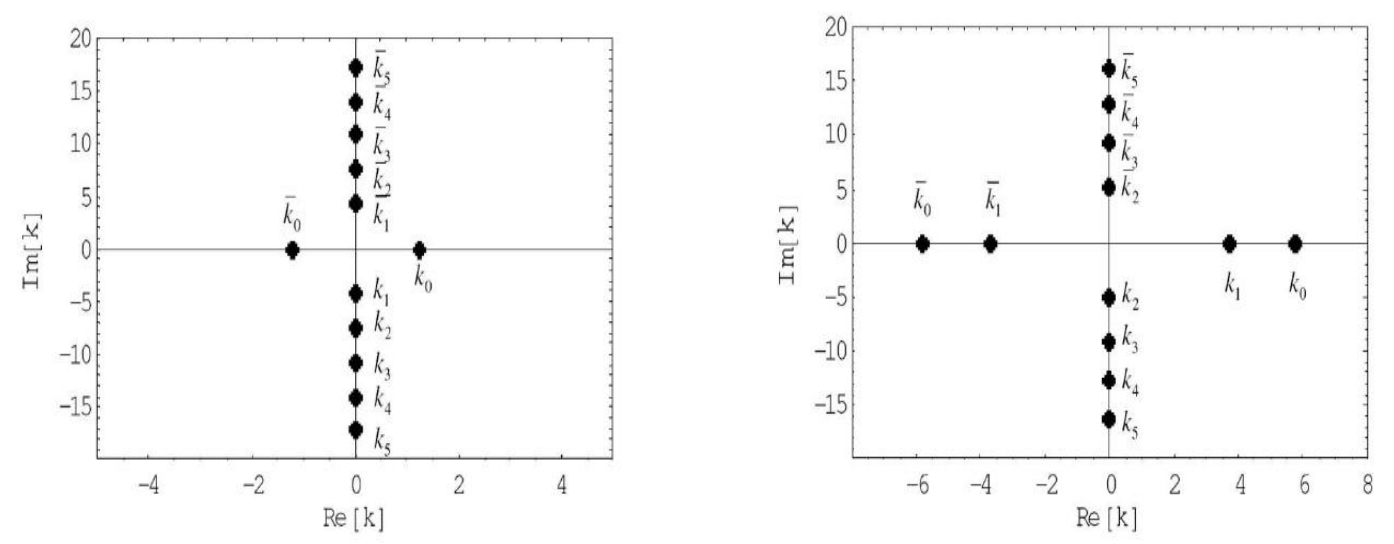

Fig.6 Analytical wave number values of undamping system at $\omega=2$. Fig.7 Analytical wave number values of undamping system at $\omega=6$.

At the same times, Figures 8(a) 10(a) are show $\omega=0.4, \omega=2$ and $\omega=6$ of the numerical wave numbers of undamping system in the complex plane, where the blue line is represent $\Phi(x, y)=0$ and the read line is represent $\Psi(x, y)=0$. Figures 8(b) 10(b) are show $\omega=0.4, \omega=2$ and $\omega=6$ of the roots solution in the undamping system in the complex plane by using numerical methods. Respectively, it is obviously to find that the analytical solution of Figures $5 \sim 7$ match well the numerical solution in Figures 8 (b) 10(b), and both analytical solutions and numerical solutions are shown in Table 2.

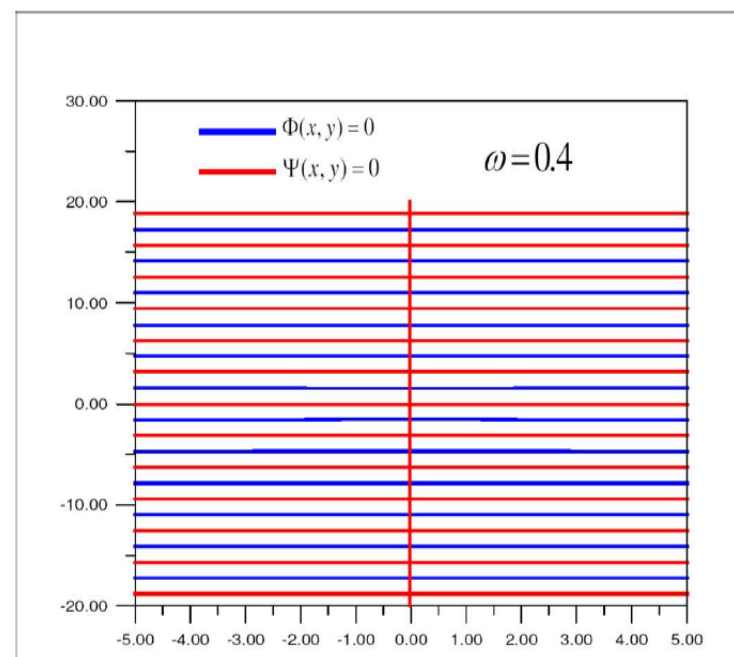

Fig.8 (a) Numerical solution of $\Phi(x, y)=0$ and $\Psi(x, y)=0$

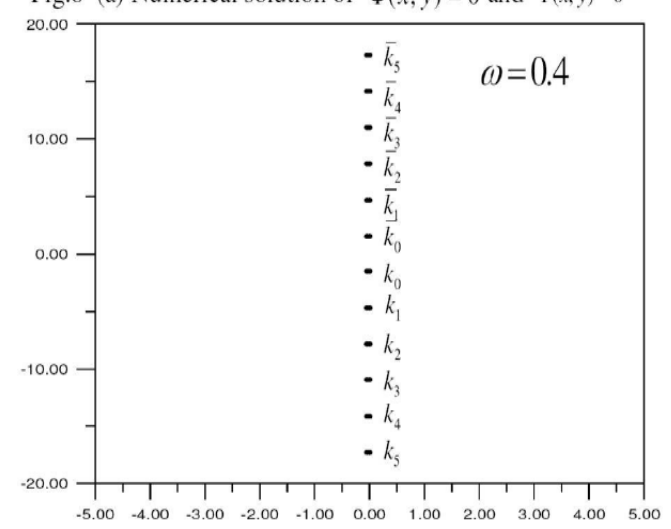

Fig.8 (b) Numerical wave number values of undamping system .

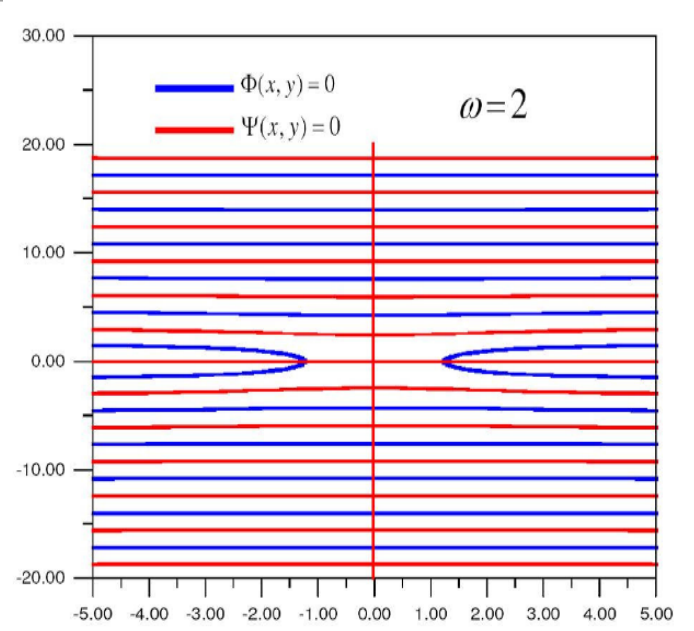

Fig.9 (a) Numerical solution of $\Phi(x, y)=0$ and $\Psi(x, y)=0$

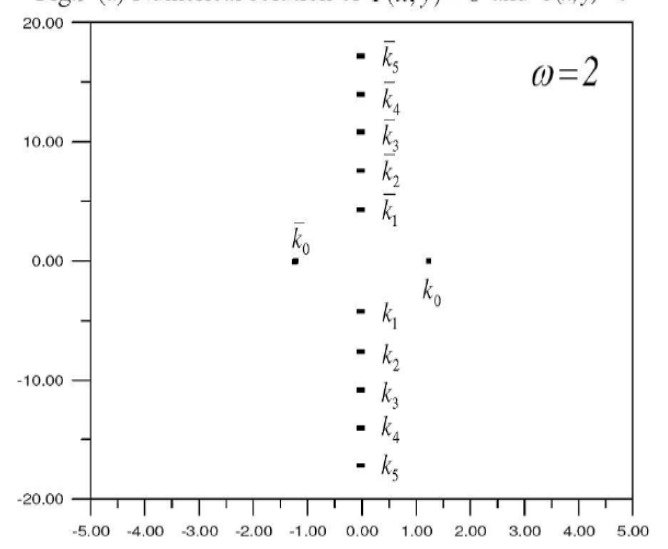

Fig.9 (b) Numerical wave number values of undamping system . 


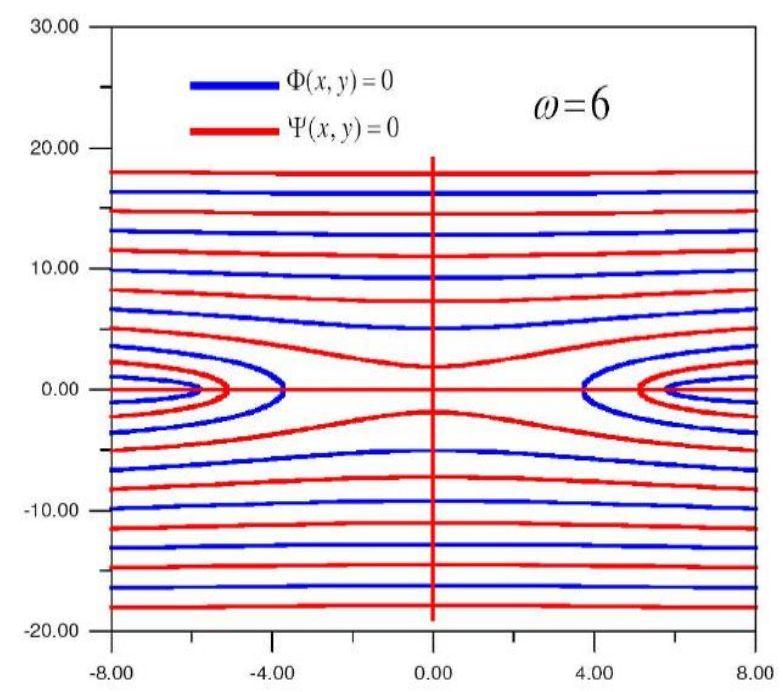

Fig.10 (a) Numerical solution of $\Phi(x, y)=0$ and $\Psi(x, y)=0$

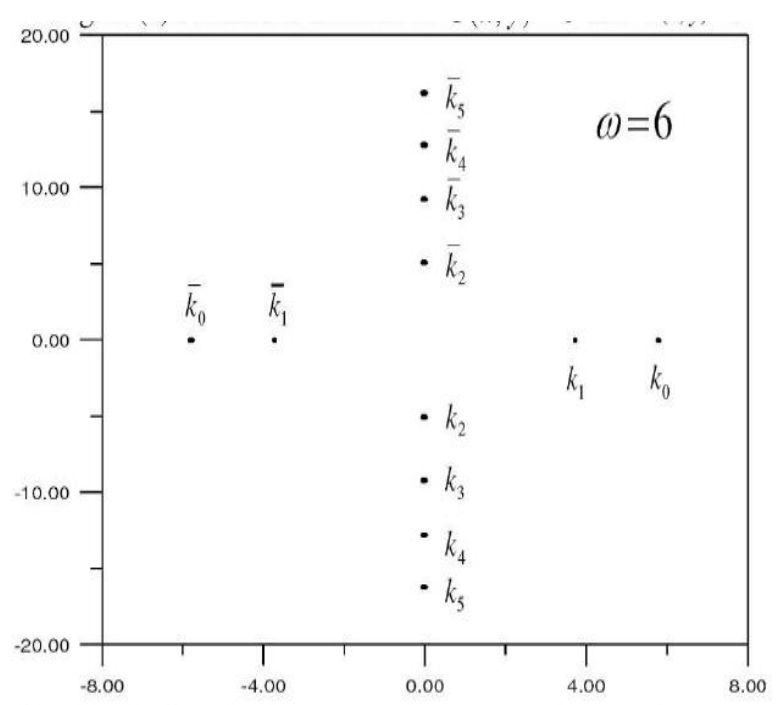

Fig.10 (b) Numerical wave number values of undamping system .

Table 2 The compare wave numbers of both analytical solution solutions and numerical solutions.

\begin{tabular}{|c|c|c|c|c|c|c|c|}
\hline Frequency & Method & $n=0$ & $n=1$ & $n=2$ & $n=3$ & $n=4$ & $n-5$ \\
\hline \multirow{2}{*}{$\omega=0.4$} & $\begin{array}{l}\text { Analytical } \\
\text { solution }\end{array}$ & $\begin{array}{c}0 \\
-1.519 \mathrm{i}\end{array}$ & $\begin{array}{c}0 \\
-4.695 i\end{array}$ & $\begin{array}{c}0 \\
-7.8437 i\end{array}$ & $\begin{array}{c}0 \\
-10.998 \mathrm{i}\end{array}$ & $\begin{array}{c}0 \\
-14.131 i\end{array}$ & $\begin{array}{c}0 \\
-17.274 \mathrm{i}\end{array}$ \\
\hline & $\begin{array}{l}\text { Numerical } \\
\text { solution }\end{array}$ & $\begin{array}{c}0 \\
-1.519 \mathrm{i}\end{array}$ & $\begin{array}{c}0 \\
-4.695 \mathrm{i}\end{array}$ & $\begin{array}{c}0 \\
-7.8437 \mathrm{i}\end{array}$ & $\begin{array}{c}0 \\
-10.998 \mathrm{i}\end{array}$ & $\begin{array}{c}0 \\
-14.131 i\end{array}$ & $\begin{array}{c}0 \\
-17.274 \mathrm{i}\end{array}$ \\
\hline \multirow{2}{*}{$\omega=2$} & $\begin{array}{l}\text { Analytical } \\
\text { solution }\end{array}$ & $\begin{array}{l}1.23789 \\
-0 i\end{array}$ & $\begin{array}{c}0 \\
-4.267 i\end{array}$ & $\begin{array}{c}0 \\
-7.5951 \mathrm{i}\end{array}$ & $\begin{array}{c}0 \\
-10.8122 \mathrm{i}\end{array}$ & $\begin{array}{c}0 \\
-13.995 i\end{array}$ & $\begin{array}{c}0 \\
-17.163 i\end{array}$ \\
\hline & $\begin{array}{l}\text { Numerical } \\
\text { solution }\end{array}$ & $\begin{array}{l}1.23789 \\
-0 i\end{array}$ & $\begin{array}{c}0 \\
-4.267 i\end{array}$ & $\begin{array}{c}0 \\
-7.5951 \mathrm{i}\end{array}$ & $\begin{array}{c}0 \\
-10.8122 \mathrm{i}\end{array}$ & $\begin{array}{c}0 \\
-13.995 i\end{array}$ & $\begin{array}{c}0 \\
-17.163 \mathrm{i}\end{array}$ \\
\hline \multirow{2}{*}{$\omega=6$} & $\begin{array}{l}\text { Analytical } \\
\text { solution }\end{array}$ & $\begin{array}{l}5.7907 \\
-0 i\end{array}$ & $\begin{array}{l}3.7139 \\
-0 \mathrm{i}\end{array}$ & $\begin{array}{c}0 \\
-5.0680 i\end{array}$ & $\begin{array}{c}0 \\
-9.2142 i\end{array}$ & $\begin{array}{c}0 \\
-12.808 \mathrm{i}\end{array}$ & $\begin{array}{c}0 \\
-16.204 i\end{array}$ \\
\hline & $\begin{array}{l}\text { Numerical } \\
\text { solution }\end{array}$ & $\begin{array}{l}5.7907 \\
.0 \mathrm{i}\end{array}$ & $\begin{array}{l}3.7139 \\
-0 \mathrm{i}\end{array}$ & $\begin{array}{c}0 \\
-5.0680 \mathrm{i}\end{array}$ & $\begin{array}{c}0 \\
-9.2142 i\end{array}$ & $\begin{array}{c}0 \\
-12.808 \mathrm{i}\end{array}$ & $\begin{array}{c}0 \\
-16.204 \mathrm{i}\end{array}$ \\
\hline
\end{tabular}

From the Table 2, we can find when the natural frequency is increasing, the low order wave numbers from imaginary part turn into real part; however, the high order wave numbers will not change a lot in imaginary part.

From the Eq.(4), the transcendental equations of Rayleigh wave numbers are many hyper cosine and hyper sine multi-product. They are not easily to find the wave numbers of Rayleigh modes by using analytical method. For Rayleigh modes, we can use the numerical method to solve the transcendental equations of Rayleigh wave numbers in Eq. (4). Figures 11 12 are shown numerical wave numbers of 0.05 hysteretic damping ratio on $\omega=0.4, \omega=2$. 


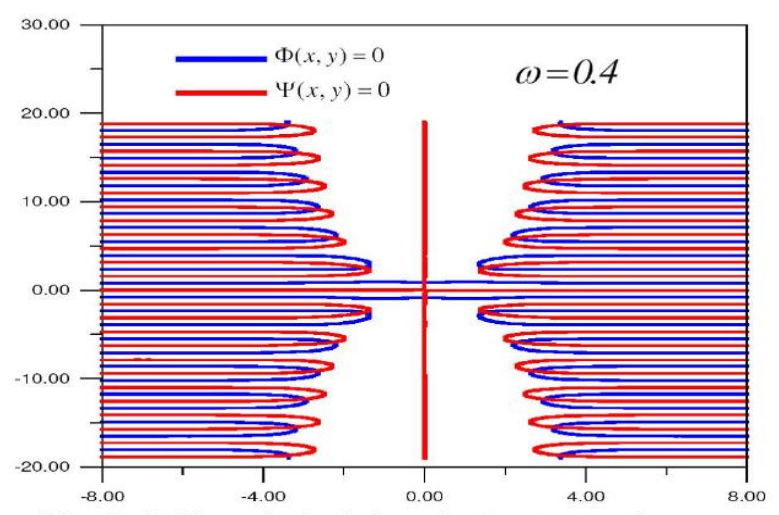

Fig. 11 (a) Numerical solution of $\Phi(x, y)=0$ and $\Psi(x, y)=0$

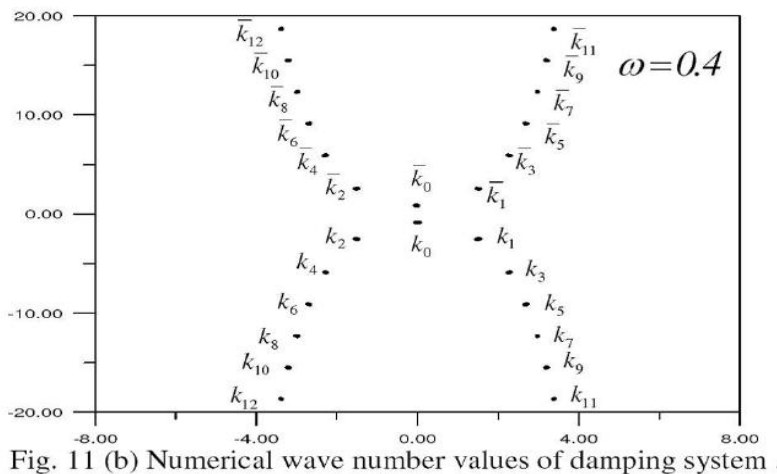

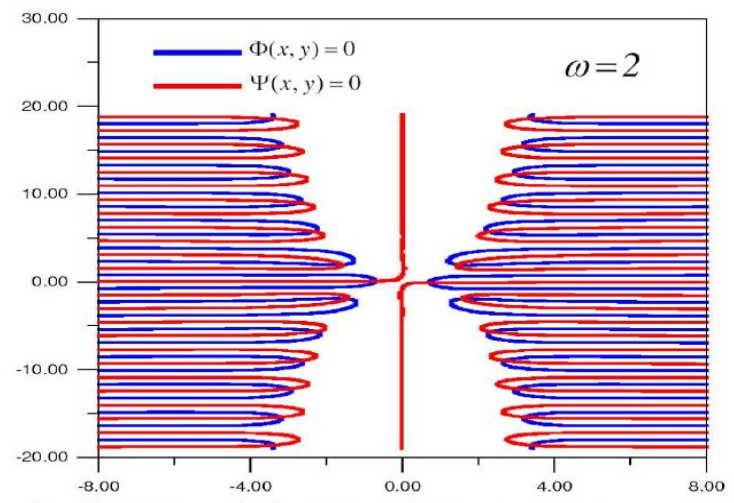

Fig. 12 (a) Numerical solution of $\Phi(x, y)=0$ and $\Psi(x, y)=0$

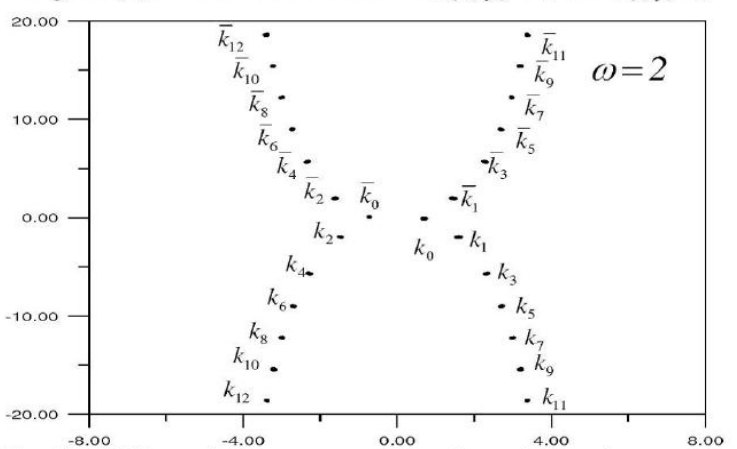

Fig. 12 (b) Numerical wave number values of damping system

In those figures, we can find the Rayleigh wave numbers have the same a pair of roots as Love modes. Consequently, all of the homogeneous solutions are applied to solve the impedance functions both in layered medium [12] and in half-space medium [16].

\section{Summary}

We have used an efficient technique to solve the complex roots in the transcendental equations. In this procedure, we have developed a complete computer program to solve the transcendental equations, and the wave numbers of transient wave propagation can be calculated numerically and analytically in Love modes; meanwhile, the Rayleigh modes can be solved by using numerical method. The mathematical model of the transient wave propagation is being used for constructing the impedance matrices of foundation embedded in layered medium.

\section{Acknowledgment}

This work is sponsored by Finance Department in Fujian of China under Contract No. 2014Y0040. The support is greatly appreciated.

\section{References}

[1] T.J. Tzong, J. Penzien, Hybrid modelling of a single-layer half-space system in soil-structure interaction, Earthquake Eng. Struct. Dyn., 1986, Vol.14(4), PP.517-530.

[2] J. Lysmer, R.L. Kuhlemeyer, Finite dynamic model for infinite media, J. Eng. Mech. Div., 1969, Vol.95(4), PP.859-877.

[3] W. White, S. Valliapan and I.K. Lee, Unified boundary for finite dynamic model, J. Eng. Mech. Div., 1977, Vol.95(5), PP.949-964.

[4] H. Werkel, Dynamic finite element analysis of three-dimensional soil models with a transmitting element, Earthquake Eng. Struct. Dyn., 1986, Vol.14(1), PP.41-60. 
[5] S. Ahmad, P.K. Banerjee, Multi-domain BEM for two-dimensional problems in elastodynamics, Int J. Num. Meth. in Eng, 1988, Vol.26(4), PP.891-911.

[6] Y.K. Chow , IM. Smith, Static and Periodic infinite solid elements, Int. J. Num. Meth. in Eng, 1981, Vol.17(4), PP.503-526.

[7] R.Y. Pak, B.B. Guzina, Seismic soil-structure interaction analysis by direct boundary element methods, International Journal of Solids and Structures, 1999, Vol.36(31), PP.4743-4766.

[8] K. Sezawa, Further studies on rayleigh waves having some azimuthal distribution. Bulletin of Earthquake Research Institute, 1929, Vol.6, PP.1-18.

[9] G.S. Liou, Analytic solutions for soil-structure interaction in layered media, Earthquake Eng. Struct. Dyn., 1989, Vol.18(5), PP.667-686.

[10] G.S. Liou, G.C. Lee and L.R. Ketter, Analytic solution for dynamic loading on half-space,. ASCE J Eng Mech., 1991, Vol.117(7), PP.1485-1495.

[11] G.S Liou, G.C. Lee, Impedance matrices for axial symmetric foundations on layered media, Struct Eng Earthquake Eng., 1992, Vol.9(1), PP.33-44.

[12] G.S. Liou, I.L. Chung, Impedance matrices for circular foundation embedded in layered medium, Soil Dyn. Earthquake Eng., 2009, Vol.29, PP.677-692.

[13] X.M. Ding, C.J. Zheng and H.L. Liu, A theoretical analysis of vertical dynamic response of large-diameter pipe piles in layered soil, J. Cent. South Univ., 2014, Vol.21, PP.3327-3337.

[14] G. Popov, N. Vaysfel'd, The torsion of the conical layered elastic cone, Acta. Mech., 2014, Vol.225, PP.67-76.

[15] M.P. Slavica, P.C. Martin and T. Ranko, On the exact analytical solution of some families of equilibrium critical thickness transcendental equations, Aip. Advences, 2014, Vol.4, PP.1-8.

[16] I.L.Chung, G.S. Liou, Calculation of impedances for axial symmetric foundation embedded in half-space medium using solutions for one layer stratum, Latin American Journal of Solids and Structures, 2013, Vol.10, PP.1225-1241. 